\title{
Otimização de arquibancadas temporárias via análise por elementos finitos
}

\author{
Ivy Jeann Pinto Marinho ${ }^{1}$, Luiz Eloy Vaz ${ }^{2}$, Roberto Leal Pimentel ${ }^{3}$
}

Trabalho recebido em 07/06/2006 e aprovado para publicação em 06/09/2006.

\section{Resumo}

Estudos de otimização aplicados a arquibancadas metálicas temporárias foram realizados utilizando-se o programa computacional ANSYS e tomando como casos de estudo duas estruturas inspecionadas. Os níveis elevados de tensão nas barras e a possibilidade de ressonância da estrutura na direção lateral foram os principais problemas identificados. O papel do sistema de contraventamento em evitar que freqüências naturais da estrutura ficassem dentro da faixa crítica de freqüências na direção lateral foi investigado, faixa esta relacionada a movimentos dos espectadores nesta direção. No processo de otimização, o nível de tensão nas barras e a freqüência natural lateral da estrutura foram definidos como variáveis de estado, as dimensões das seções transversais das barras como variáveis de projeto e a massa total da estrutura como a função objetivo. Concluiu-se que uma das arquibancadas, apesar de não apresentar problemas de potencial ressonância na direção lateral, possuía barras com seção transversal subdimensionadas para resistir a ações estáticas. A segunda arquibancada, além de apresentar barras subdimensionadas para resistir a ações estáticas, apresentava potencial ressonância na direção lateral. Neste caso, o projeto ótimo só foi atingido após o rearranjo do sistema de contraventamento.

Palavras-chave. arquibancadas temporárias, otimização, vibração, contraventamento.

1 Prof. MSc., Departamento de Engenharia Civil, PUC-Rio. Endereco atual: Praça Epitácio Pessoa, 35, Centro, Itabaiana, PB, Brasil, CEP 58.360-000. E-mail: ivymarinho@yahoo.com.br

2 Prof. Dr. Ing, Departamento de Mecânica Aplicada e Estruturas, Escola Politécnica da UFRJ. Prédio do Centro de Tecnologia, Bloco D, 2º andar, sala 203, Cidade Universitária, Rio de Janeiro, RJ, Brasil, CEP 21945-970. E-mail: eloy@poli.ufrj.br

3 Prof. PhD, Departamento de Engenharia Civil, Universidade Federal da Paraíba. Campus I, Centro de Tecnologia, CEP 58051-900, João Pessoa, PB, Brasil. Fone: +55 83 3216-7355. Fax: +55 83 3216-7179. E-mail:r.pimentel@uol.com.br 


\section{Introdução}

Arquibancadas metálicas temporárias são estruturas compostas por barras e conectores, sendo montadas in loco, para uso principalmente em eventos públicos. Estas estruturas estão sujeitas a cargas estáticas e dinâmicas, estas últimas causadas pelo movimento dos espectadores.

Têm ocorrido falhas nestas estruturas, incluindo o colapso total, como o ocorrido na Córsega em maio de 1992, com dezessete mortos e mais de 2500 feridos (Ji e Ellis 1997). Outro caso ocorreu em 1994 em Londres, onde 40 pessoas foram gravemente feridas (Ji e Ellis 1999). No Brasil, também há casos de acidentes com palanques e arquibancadas temporárias, como o ocorrido em Grajaú (SP) em 1995 com 50 feridos, em uma estrutura com capacidade para 300 espectadores, ou mais recentemente em 2002, em um rodeio em São Paulo, onde 80 pessoas ficaram feridas após o colapso parcial da arquibancada.

Inspeções realizadas em várias arquibancadas temporárias mostraram que os elementos estruturais e disposição das barras de tais estruturas variam razoavelmente e que aparentemente não há regras para dispor o sistema de contraventamento, dada a variabilidade observada. O principal problema de vibração nestas estruturas é o da possibilidade de ressonância na direção lateral, devido aos movimentos sincronizados dos espectadores nesta direção (Littler 1996). O papel das barras de contraventamento é ressaltado nesta questão, devendo ser colocadas de modo a contribuir para que a estrutura apresente freqüências naturais fora da faixa de freqüência crítica de harmônicos da excitação (Ji e Ellis 1997; Marinho 2002).

Neste trabalho, apresentam-se procedimentos para o dimensionamento ótimo e um estudo de caso de arquibancadas metálicas temporárias, sendo examinados aspectos relacionados aos projetos estático e dinâmico. O uso de rotinas de otimização é explorado, levando-se em consideração restrição no valor da freqüência fundamental da estrutura para evitar problemas de ressonância. O objetivo é alcançar um projeto ótimo, através da otimização das seções transversais dos elementos estruturais e minimização da massa total da estrutura.

Duas arquibancadas foram inspecionadas e tomadas como casos de estudo, sendo aqui denominadas MC1 e AR1. As estruturas foram modeladas utilizando-se o programa computacional ANSYS. Análises estáticas foram realizadas visando identificar elementos estruturais com possíveis problemas de dimensionamento. Análises modais foram subseqüentemente realizadas, fornecendo valores das freqüências naturais e modos de vibração das estruturas e permitindo a identificação de modos críticos relacionados à vibração na direção lateral. Em seguida, ambas as estruturas foram otimizadas, aplicando algoritmo disponível no programa ANSYS, definindo-se a freqüência fundamental da estrutura na direção lateral e os níveis de tensão nas barras como variáveis de estado, as dimensões das seções transversais dos elementos como variáveis de projeto e a massa total da estrutura como a função objetivo. Paralelamente à otimização da arquibancada AR1, regras para uma adequada disposição dos elementos 
do sistema estrutural de contraventamento foram discutidas e seus efeitos na obtenção de um projeto ótimo analisados.

\section{Descrição e análise das estruturas selecionadas}

As arquibancadas investigadas estão apresentadas na Fig. 1. Especificações técnicas, modelagens em elementos finitos e análises estática e modal para obtenção dos níveis de tensões, freqüências naturais e modos de vibração das estruturas inspecionadas são a seguir apresentados.

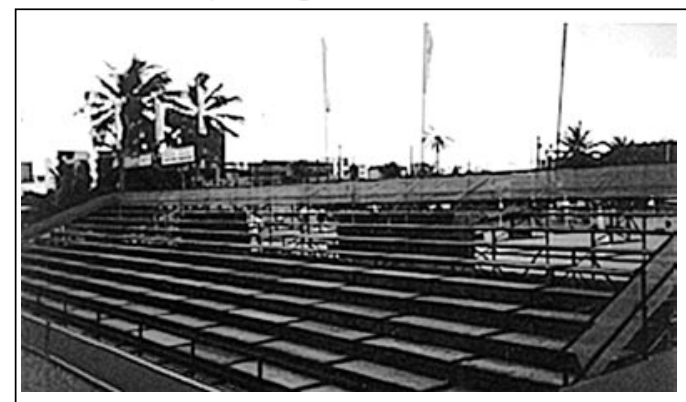

a) $\mathrm{MC} 1$

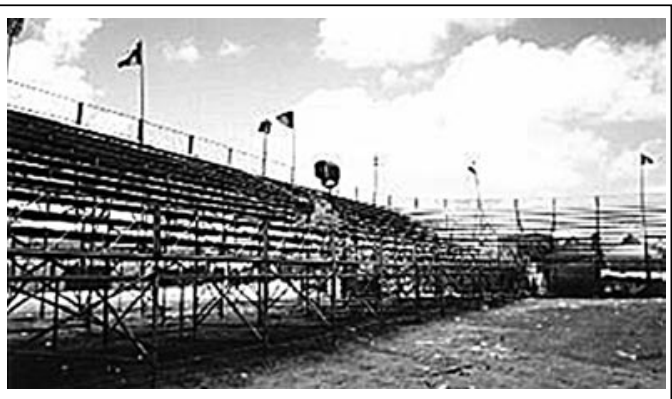

b) AR1

Figura 1: Arquibancadas estudadas

\subsection{Especificações técnicas das estruturas e modelagem}

Ambas as estruturas eram formadas for quadros tridimensionais e barras metálicas tubulares de seção transversal e comprimento padronizados, encontrando-se simplesmente apoiadas no solo (Fig. 1). Os modelos numéricos em elementos finitos desenvolvidos para as estruturas estão apresentados na Fig. 2, sendo compostos cada um por 16 ou 12 módulos. Este número de módulos foi definido por constituir uma unidade para cada estrutura, do ponto de vista de colocação de barras de contraventamento. $\mathrm{O}$ módulo de cada arquibancada é descrito a seguir:

- Para a estrutura MC1, o módulo é composto por 3 tablados de madeira, sendo cada tablado com 2,10 m de comprimento por 0,6 m de largura e capacidade indicada para 4 ocupantes, apoiados em uma estrutura aporticada designada pelos fabricantes por "cavalo", este com 2,42 m de comprimento e altura de 1,20 m. A estrutura modelada com os 16 módulos apresenta 8,40 m de comprimento, 9,68 m de largura, altura de 4,80 m e capacidade para 192 ocupantes (Fig. 2).

- Para a estrutura AR1, o módulo é composto por 4 tablados de madeira (sendo o módulo do topo composto por 5), com cada tablado tendo capacidade indicada para 5 ocupantes e com 2,30 m de comprimento por 0,7 m de largura, apoiados em um 
"cavalo", este com 2,30 m de comprimento e altura de 1,20 m. A estrutura modelada com 12 módulos apresenta 9,20 m de comprimento, 6,90 m de largura, altura de 4,80 m e capacidade para 240 ocupantes (Fig. 2).

As principais propriedades dos materiais e as dimensões medidas dos componentes das estruturas estão apresentados na Tabela 1.

Tabela 1: Dimensões dos componentes e propriedades dos materiais.

\begin{tabular}{cccc}
\hline \multirow{2}{*}{ Características } & MC1 & AR1 & MC1/AR1 \\
\cline { 2 - 4 } & & Aço & Madeira \\
\hline $\mathrm{d}_{1}(\mathrm{~mm})$ & 38,10 & 30,80 & - \\
$\mathrm{e}_{1}(\mathrm{~mm})$ & 3,0 & 3,0 & - \\
$\mathrm{d}_{2}(\mathrm{~mm})$ & 31,73 & 3,0 & - \\
$\mathrm{e}_{2}(\mathrm{~mm})$ & 3,0 & 31,75 & - \\
$\mathrm{d}_{3}(\mathrm{~mm})$ & - & 3,0 & - \\
$\mathrm{e}_{3}(\mathrm{~mm})$ & - & 12,70 & - \\
$\mathrm{d}_{4}(\mathrm{~mm})$ & - & 1,5 & - \\
$\mathrm{e}_{4}(\mathrm{~mm})$ & - & - & 20 \\
$\mathrm{e}_{\mathrm{b}}(\mathrm{mm})$ & - & 250 & - \\
$\mathrm{f}_{\mathrm{y}}(\mathrm{MPa})$ & 250 & 210 & 22,74 \\
$\mathrm{E}(\mathrm{GPa})$ & 210 & 0,3 & 0,2 \\
$v$ & 0,3 & 7850 & 1143 \\
\hline $\mathrm{m}_{\text {esp }}\left(\mathrm{kg} / \mathrm{m}^{3}\right)$ & 7850 & & \\
\hline
\end{tabular}

Na Tabela 1, $d_{i}$ é e são, respectivamente, o diâmetro externo e a espessura da barra tubular vazada do tipo $i ; f_{y}, E, v$ e $m_{\text {esp }}$ são, respectivamente, a tensão de escoamento, o módulo de elasticidade, o coeficiente de Poisson e a massa específica. Já e é a espessura dos tablados, mantida constante no processo de otimização. As propriedades dos materiais foram definidas a partir de valores de referência para cada material (NBR 8800/1986; NBR 7190/1997).

Dois tipos de elementos finitos foram usados na modelagem: para as barras metálicas tubulares foi empregado o PIPE16 (ANSYS 2001). Este elemento tem seis graus de liberdade em cada um dos dois nós, sendo assemelhado a um elemento de viga tridimensional, mas com simplificações devido a sua simetria e geometria tubular. Já para os tablados utilizou-se o elemento de casca SHELL63 (ANSYS 2001). Este elemento também apresenta seis graus de liberdade em cada um dos quatro nós. Os modelos em elementos finitos tinham inicialmente 734 elementos e 380 nós para a arquibancada MC1 e 950 elementos e 530 nós para a AR1. 


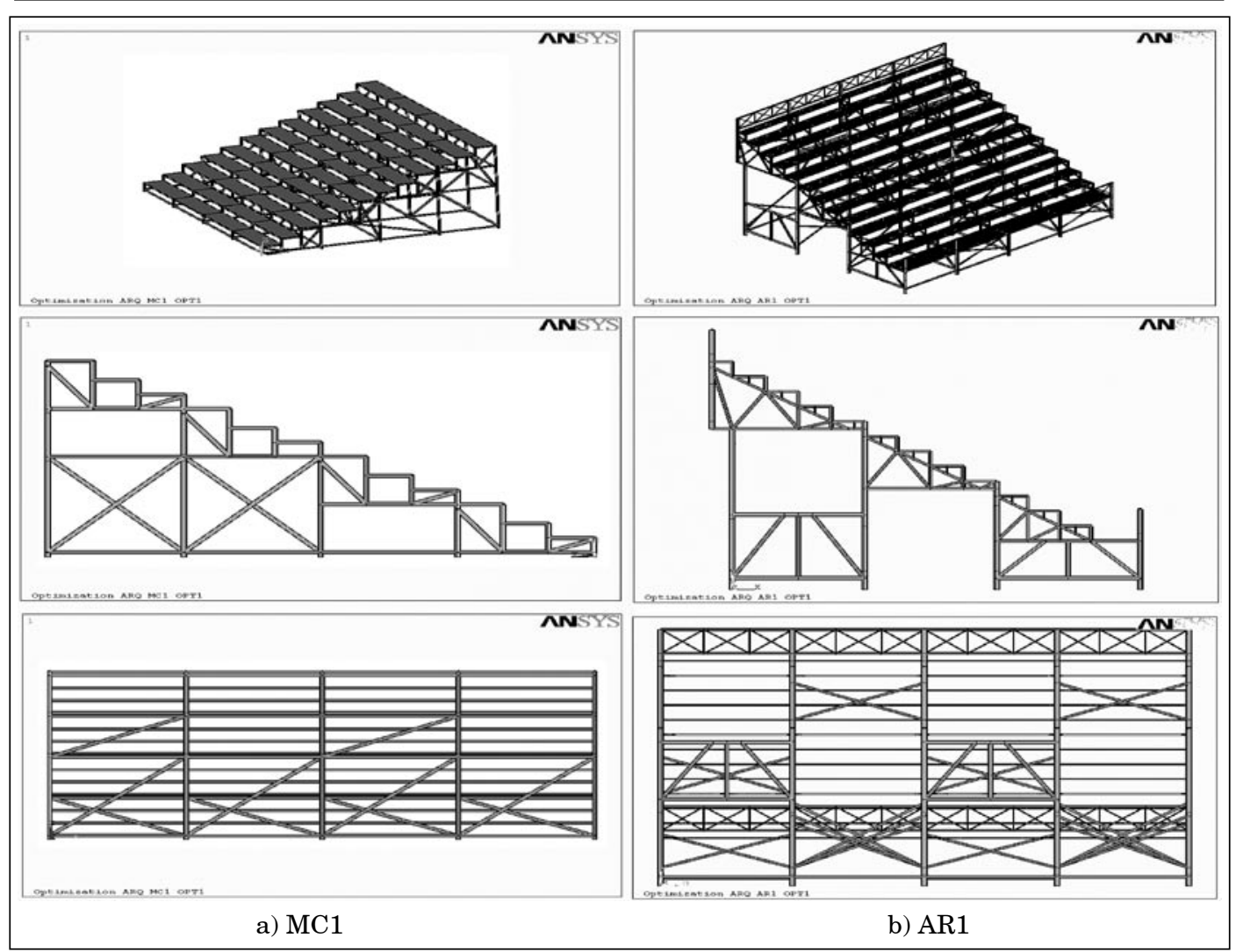

Figura 2: Modelos: vistas em perspectiva, lateral, e fundo.

\subsection{Análises}

Análise Estática: realizou-se cálculo de verificação, levando-se em consideração o disposto nas normas NBR 6120/80 (1980), que estabelece carregamento estático de 4 $\mathrm{kN} / \mathrm{m}^{2}$ para este tipo de estrutura e na NBR 8800/86 (1986). Os seguintes estados limites foram considerados no cálculo: flambagem elástica, flambagem plástica, flambagem local das chapas e esmagamento com escoamento total da seção.

Tais considerações resultaram em tensões limites de projeto para as barras de 46,35 MPa para a arquibancada MC1 e 94,9 MPa para a arquibancada AR1. Estes valores foram determinados após análise de tensões nas várias barras de cada arquibancada. Entretanto, os esforços atuantes calculados em um número significativo de barras analisadas, considerando-se os diferentes comprimentos e diâmetros, foram consideravelmente superiores à capacidade resistente das mesmas. Em várias barras, tensões acima de $66 \mathrm{MPa}$ nas barras da MC1 e $163 \mathrm{MPa}$ nas barras da AR1 foram atingidas.

Análise Modal: Os valores das freqüências naturais dos cinco primeiros modos de vibração, bem como sua identificação, podem ser observados nas Figs. 3 e 4. 
A freqüência fundamental foi de $5,25 \mathrm{~Hz}$ para a arquibancada $\mathrm{MC} 1$, relacionada a um modo de vibração com movimentos de frente para trás (Fig. 3), e de $2,36 \mathrm{~Hz}$ para a estrutura AR1, relacionada a um modo de vibração lateral (Fig. 4). Littler (1996) apresentou testes em quarenta arquibancadas metálicas, encontrando freqüências naturais relacionadas a modos laterais na faixa de 1,8 a $6,0 \mathrm{~Hz}$ para estruturas vazias. Este mesmo autor sugeriu que, ao se projetar uma arquibancada metálica reutilizável, considerando a arquibancada vazia, deve-se buscar uma freqüência natural nesta direção maior que $4,0 \mathrm{~Hz}$ para evitar problemas de ressonância. Tal valor situaria a estrutura fora da faixa de freqüências relacionadas à excitação lateral provocada por movimentos sincronizados de indivíduos, que vai de $1,5 \mathrm{~Hz}$ a $3,5 \mathrm{~Hz}$. A consideração de arquibancadas vazias decorre do fato de estudos mostrarem que com a estrutura ocupada há mudanças nas freqüências naturais, além de ocorrerem variações nestas durante 0 evento, impedindo uma correta quantificação dos valores (Littler 1996). Dessa forma, a arquibancada MC1 não apresentou freqüências laterais dentro da faixa crítica de ressonância enquanto que a arquibancada AR1 estaria com freqüência lateral inferior a $4,0 \mathrm{~Hz}$, sugerindo um potencial problema de vibração excessiva nesta direção.

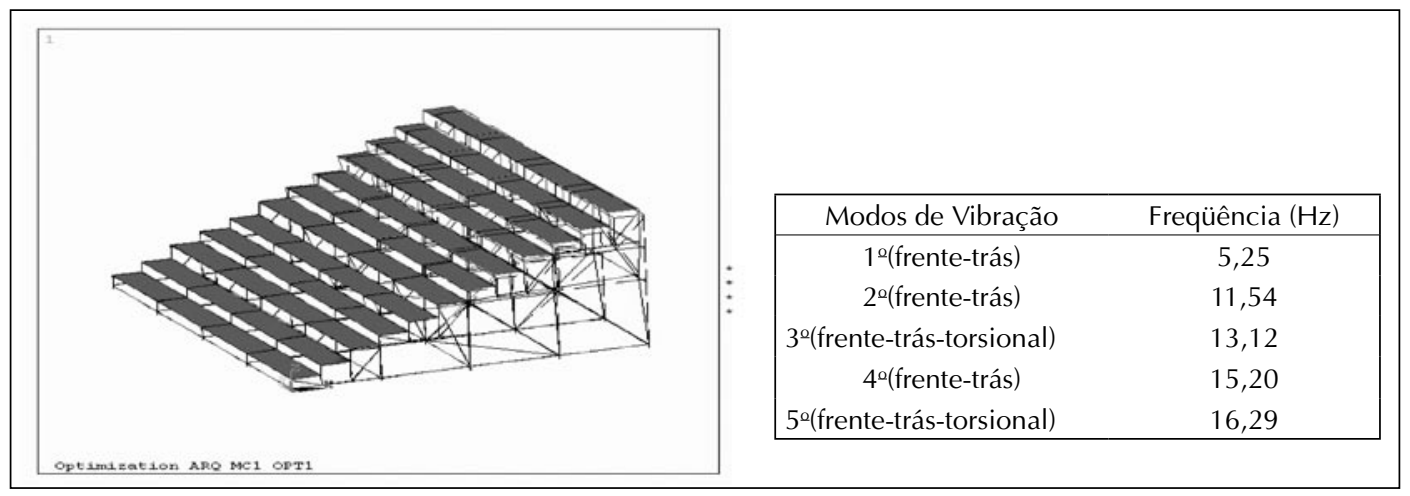

Figura 3: MC1-Freqüências naturais e primeiro modo de vibração, direção frente-trás.

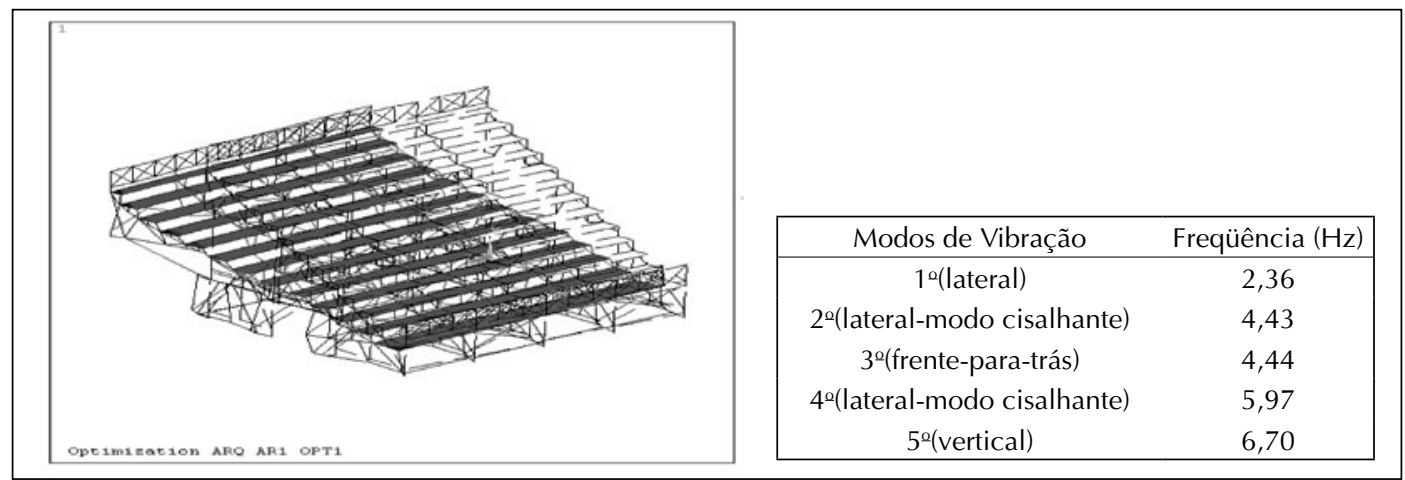

Figura 4: AR1-Freqüências naturais e primeiro modo de vibração na direção lateral. 


\section{O método de otimização}

O método de Primeira Ordem, presente no programa ANSYS, foi o utilizado na otimização das estruturas de arquibancada. Segundo a documentação do programa (ANSYS 2001), este método produz bons resultados para problemas onde as variáveis dependentes variam amplamente em uma extensa faixa de espaço de projeto. Por outro lado, resultados obtidos aplicando-se este método a estruturas reticuladas presentes na literatura foram promissores (Marinho 2002), justificando a sua escolha. No processo de otimização, minimiza-se ou maximiza-se a função objetivo do problema. Três tipos de parâmetros caracterizam o processo de otimização: variáveis de projeto, variáveis de estado e a função objetivo (Haftka e Gurdal 1993).

\subsection{Definição dos parâmetros de otimização}

Os seguintes parâmetros foram definidos: 1. variáveis de projeto, definidas como 0 diâmetro e espessura dos tubos metálicos. Estas são quantidades independentes dentro de faixas de restrições especificadas e que variam durante o processo de otimização, tendo limites superiores e inferiores especificados que servem como restrições; 2. Variáveis de estado, quantidades que fixam as restrições de projeto dentro de limites máximo e mínimo, neste caso os valores de tensão e freqüência fundamental. Estas também são conhecidas como variáveis dependentes. O método de otimização adotado utiliza a informação dos gradientes destas variáveis em relação às variáveis de projeto; e 3. função objetivo, ou seja, função que se quer otimizar, no caso, a massa total da estrutura. O problema é matematicamente representado por:

$$
\mathrm{x}=\left[\mathrm{x}_{1}, \mathrm{x}_{2}, \mathrm{x}_{3} \ldots \mathrm{x}_{\mathrm{n}}\right]
$$

Na Eq. 1, xi são as variáveis de projeto, sujeitas a n restrições com limites superiores e inferiores,

$$
\underline{\mathrm{x}}_{\mathrm{i}} \leq \mathrm{x}_{\mathrm{i}} \leq \overline{\mathrm{x}}_{\mathrm{i}} \quad(i=1,2,3, \ldots, \mathrm{n})
$$

onde $\mathrm{n}$ é o número de variáveis.

As restrições das variáveis de projeto são freqüentemente chamadas de restrições laterais e definem o que é geralmente identificado como espaço viável de projeto. Podese, então, minimizar,

$$
f=f(x)
$$

Sujeita à

$$
\begin{gathered}
g_{j}(x) \leq \overline{g_{j}} \quad\left(j=1,2,3, \ldots, m_{1}\right) \\
\underline{w_{j}} \leq w_{j}(x) \leq \overline{w_{j}} \quad\left(j=1,2,3, \ldots, m_{2}\right)
\end{gathered}
$$


Nas equações acima, f é a função objetivo, $g_{i}$ e $w_{i}$ são as variáveis de estado, com as sublinhas e sobrelinhas representando, respectivamente, os limites inferiores e superiores, e $\mathrm{m}_{1}$ e $\mathrm{m}_{2}$ são o número de restrições das variáveis de estado. Na formulação apresentada, $g_{i}(x)$ é uma variável de estado representando a tensão, que possui apenas limite superior, ao passo que $w_{i}(x)$ representa a freqüência fundamental, que possui limite inferior. Nesta última função, é necessário definir um limite superior, em virtude da metodologia utilizada pelo software na otimização.

\subsection{O método de primeira Ordem empregado pelo ANSYS}

A formulação detalhada do método está apresentada em Marinho (2002). Em síntese, o problema com restrição, expresso nas Eqs. (1) a (5), é transformado em um problema sem restrição, redefinindo-se a função objetivo mediante a introdução de funções de penalidade, que são aplicadas às variáveis de projeto e de estado.

Para cada iteração (j) no processo de otimização, um vetor de direção de busca $\mathbf{d}^{(j)}$ é criado. Na próxima iteração $(j+1)$, o vetor de direção de busca $\mathbf{d}^{(j)}$ sofre modificações de modo que em cada iteração as variáveis são atualizadas. A direção de busca é o gradiente da função objetivo sem restrição multiplicado por (-1), sendo na iteração inicial $(j=0)$ este método de busca denominado de descida íngreme. Ao aproximar-se da solução, o método tende a repetir várias vezes a mesma direção de busca. Para evitar que isto ocorra, faz-se uso do procedimento de rotação da direção do gradiente e, para iterações subseqüentes, direções conjugadas são formadas de acordo com a fórmula de recursão de Polak-Ribiere (More e Wright 1993).

Para verificar a convergência, compara-se o conjunto do projeto da iteração atual (j) com o projeto prévio (j-1) e com o melhor conjunto (b) de projeto, isto é, com o(s) melhor(es) valor(es)/resultado(s) obtido(s) até o momento considerando-se as restrições do problema, Eqs. (6) e (7).

$$
\begin{aligned}
& \left|\mathrm{f}^{(\mathrm{j})}-\mathrm{f}^{(\mathrm{j}-1)}\right| \leq \tau \\
& \left|\mathrm{f}^{(\mathrm{j})}-\mathrm{f}^{(\mathrm{b})}\right| \leq \tau
\end{aligned}
$$

Nas Eqs. (6) e (7), $\tau$ é a tolerância da função objetivo, definida pelo projetista. Caso o valor da tolerância não seja especificado pelo projetista, o ANSYS adotará um valor automático correspondente a $1 \%$ da função objetivo. $O$ término da execução também ocorrerá se o número de iterações atingir um valor limite, que também é definido pelo projetista.

O ANSYS possui um módulo de otimização no qual o procedimento aqui apresentado é implementado, sendo porém necessário realizar previamente análises estática e dinâmica (modal). Para tal implementação, utiliza-se linguagem de programação APDL (Ansys Parametric Design Language) do próprio software ou, de forma interativa, o usuário pode fornecer as informações solicitadas para o procedimento de otimização mediante o preenchimento de dados em telas apresentadas no ambiente de trabalho do software. 


\section{Otimização das arquibancadas metálicas temporárias}

O objetivo inicial era reduzir a massa da estrutura com o processo de otimização. Porém, como foi previamente discutido, a situação dos projetos atuais foi o oposto, com ambas as estruturas investigadas apresentando problemas de subdimensionamento. Além disso, no caso da arquibancada AR1, a freqüência fundamental estava abaixo do limite mínimo recomendado, situando-se dentro da faixa crítica passível de ressonância. Deste modo, com o processo de otimização deve-se corrigir as seções transversais das barras e elevar o valor da freqüência fundamental.

\subsection{Otimização da arquibancada $\mathrm{MCl}$}

Para a arquibancada MC1, apenas o projeto estático foi objeto dos procedimentos de otimização. Foram obtidos valores adequados para as seções transversais dos elementos estruturais e o nível de tensão foi mantido dentro dos limites de projeto. Com relação às freqüências naturais, não havia modos laterais com freqüência abaixo de $4,0 \mathrm{~Hz}$, assim permanecendo após a otimização. Foi observado que peso da estrutura metálica aumentou, logicamente para se adequar ao nível de tensão pretendido nos elementos estruturais, conforme já discutido.

\subsubsection{Modificações propostas na estrutura e otimização final - MC1}

As áreas das seções transversais dos tubos que antes apresentavam valores de 3,3 $\mathrm{cm}^{2}$ e $2,7 \mathrm{~cm}^{2}$, após a otimização passaram a apresentar os valores de $4,8 \mathrm{~cm}^{2} \mathrm{e} 1,6 \mathrm{~cm}^{2}$. Quanto à freqüência natural fundamental, não foi enfoque da otimização, variando de $5,25 \mathrm{~Hz}$ para $5,33 \mathrm{~Hz}$ após a otimização, sem alteração no modo de vibração associado à mesma. Os elementos de madeira tinham inicialmente $1382,6 \mathrm{~kg}$ de massa e a estrutura de aço $1471,5 \mathrm{~kg}$, perfazendo um total de $2854,1 \mathrm{~kg}$. Os valores iniciais, os limites de projeto (incluindo valores comercialmente disponíveis) e os valores ótimos obtidos estão apresentados na Tabela 2.

Tabela 2: Valores iniciais, valores limites e ótimos - MC1

\begin{tabular}{ccccc}
\hline Variáveis de Projeto/ & \multirow{2}{*}{ Valores iniciais } & \multicolumn{2}{c}{ Limites } & \multirow{2}{*}{ Valores ótimos } \\
\cline { 3 - 4 } & & Valor mínimo & Valor máximo & \\
\hline $\mathrm{d}_{1}(\mathrm{~mm})$ & 38,10 & 25,40 & 63,50 & 44,45 \\
$\mathrm{e}_{1}(\mathrm{~mm})$ & 3,0 & 2,0 & 4,75 & 3,75 \\
$\mathrm{~d}_{2}(\mathrm{~mm})$ & 31,73 & 25,40 & 50,80 & 25,40 \\
$\mathrm{e}_{2}(\mathrm{~mm})$ & 3,0 & 2,0 & 3,75 & 2,25 \\
$\mathrm{e}_{\mathrm{b}}(\mathrm{mm})$ & 20 & - & - & 20 \\
SMAX $(\mathrm{MPa})$ & 66,33 & - & 46,5 & 44,02 \\
FREQ $(\mathrm{Hz})$ & 5,25 & 4 & 7 & 5,33 \\
Massa total $(\mathrm{kg})$ & 2854,1 & - & - & 3151 \\
\hline
\end{tabular}


Na Tab. 2, SMAX e FREQ representam, respectivamente, os limites de tensão nas barras e freqüência fundamental da estrutura, restrições estas correlacionadas às Eqs. (4) e (5). Cabe ressaltar que o limite superior de $7 \mathrm{~Hz}$ na freqüência fundamental foi definido apenas para atender à exigência do algoritmo de otimização e que o valor da tensão máxima que orientou o projeto foi obtido com base em análise estática preliminar da estrutura no ANSYS. Conclui-se para esta análise que a otimização teve como enfoque o dimensionamento estático.

\subsection{Otimização inicial da arquibancada AR1}

Esta estrutura apresentava freqüência natural na direção lateral dentro de faixa crítica passível de ressonância. Deste modo, o processo de otimização deveria corrigir as seções transversais das barras e elevar o valor da freqüência natural, objetivando um peso ótimo para a estrutura. Após executados os procedimentos de otimização, obteve-se uma solução que satisfez as restrições de tensões, porém não atingiu a faixa de freqüência ótima de projeto. Concluiu-se que o sistema estrutural da arquibancada não permitiu alcançar um projeto ótimo. Tal fato sugeriu que o projeto inicial não comportaria a otimização com restrição de freqüência, ou seja, para o problema em questão, tinha-se um projeto "impossível" de ser otimizado dentro das restrições impostas e variáveis de projeto disponíveis. Estudos subseqüentes (Marinho 2002) revelaram que o sistema de contraventamento poderia ser a chave para encontrar a solução do problema, pois verificou-se que, além de ser relevante nas análises estáticas, possui importância fundamental nas características dinâmicas das arquibancadas reutilizáveis.

Ji e Ellis (1997) discutiram regras de disposição do sistema de contraventamento em arquibancadas temporárias. Baseados em estudos para pórticos planos, eles propuseram cinco critérios para a disposição, inclusão e/ou rearranjo das barras do sistema de contraventamento, visando um aumento de rigidez e, por conseguinte, um aumento da freqüência fundamental na direção lateral. Os cinco critérios propostos por estes autores para estruturas reticuladas planas foram os seguintes:

1. As barras de contraventamento devem ser conectadas em pavimentos diferentes, sempre do topo para a base da arquibancada;

2. As barras de contraventamento de pavimentos diferentes devem, se possível, serem conectadas diretamente;

3. As barras de contraventamento devem compor um alinhamento direto sempre que possível;

4. Barras de contraventamento de vãos adjacentes devem unir-se diretamente quando possível;

5. Caso sejam adicionadas barras de contraventamento ao sistema, sua disposição deve seguir os quatros critérios anteriores. 
Como mencionaram Ji e Ellis (1997), dois conceitos foram empregados na concepção destes critérios: caminho direto de força e distribuição uniforme de força. Os exemplos utilizados por Ji e Ellis (1997) para aplicar os conceitos propostos foram pórticos planos de grandes estruturas de arquibancadas. A aplicação progressiva dos primeiros quatro critérios foi investigada em pórticos tridimensionais em forma de degrau de arquibancada (Fig. 5), observando-se o efeito no ganho de rigidez e conseqüente aumento de freqüência. Verificou-se que os critérios, desenvolvidos para pórticos planos, foram também efetivos para pórticos tridimensionais no que diz respeito a elevar a freqüência fundamental da estrutura, como mostrado na Tabela 3.

Tabela 3: Freqüências naturais de modelo 3D, para aplicação progressiva dos critérios de contraventamento.

\begin{tabular}{|c|c|c|c|c|c|}
\hline \multirow{2}{*}{ Casos } & \multicolumn{5}{|c|}{ Freqüências Naturais $(\mathrm{Hz})$} \\
\hline & $1^{\circ} \underline{0}$ & $2^{\mathrm{o}}$ & $3^{\mathrm{o}}$ & $4^{\circ}$ & $5^{\circ}$ \\
\hline a. & 52,28 & 90,66 & 92,59 & 103,42 & 192,21 \\
\hline b. & 59,92 & 87,69 & 101,14 & 113,21 & 190,67 \\
\hline c. & 61,11 & 90,86 & 96,61 & 108,58 & 187,37 \\
\hline d. & 62,02 & 94,85 & 97,11 & 123,64 & 204,02 \\
\hline
\end{tabular}

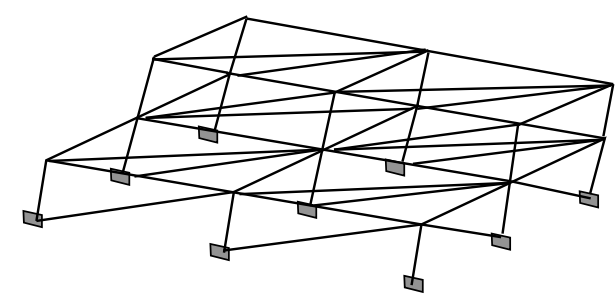

a) 1. Contraventamento topo para base.

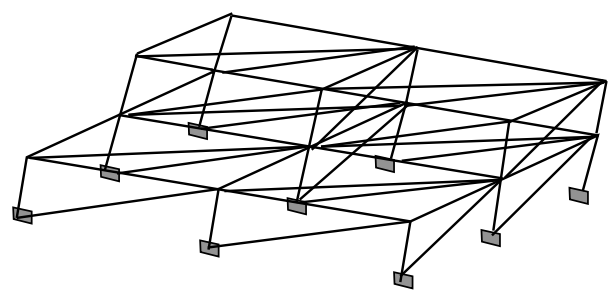

c) 3. Contraventamento em alinhamento direto.

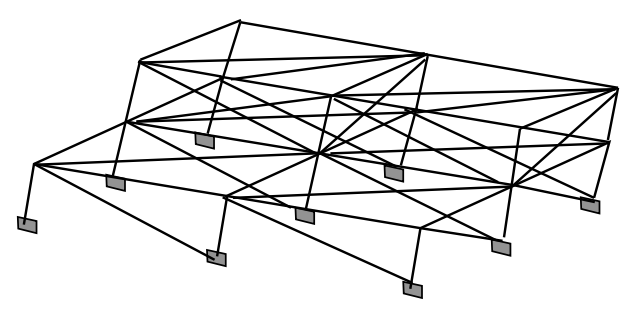

b) 2. União direta entre pavimentos.

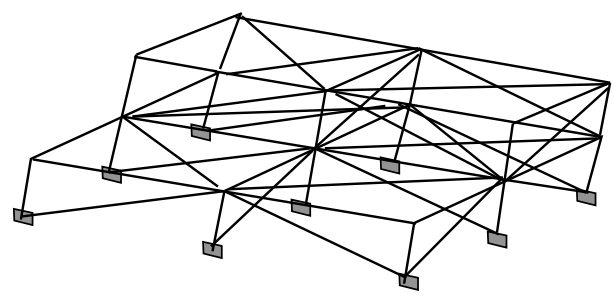

d) 4. União direta entre vãos adjacentes.

Figura 5: Critérios de Ji e Ellis aplicado a modelo 3D.

A fim de viabilizar o processo de otimização da arquibancada AR1, decidiu-se aplicar os critérios propostos, inclusive o quinto (adição de barras ao sistema de contraventamento), pois apesar de tal critério ser aparentemente anti-econômico, poderia ser necessário para atingir os objetivos da análise. 


\subsubsection{Alterações propostas na estrutura AR1}

O sistema de contraventamento da arquibancada AR1 foi reprojetado, seguindo os cinco critérios mencionados anteriormente. $\mathrm{O}$ sistema de contraventamento otimizado conforme tais critérios pode ser visto na Figura 6, sendo oportuno compará-lo ao sistema original (apresentado na Fig. 2). Após as alterações, a freqüência fundamental (na direção lateral) mudou, passando de $2,36 \mathrm{~Hz}$ para aproximadamente $3,5 \mathrm{~Hz}$.

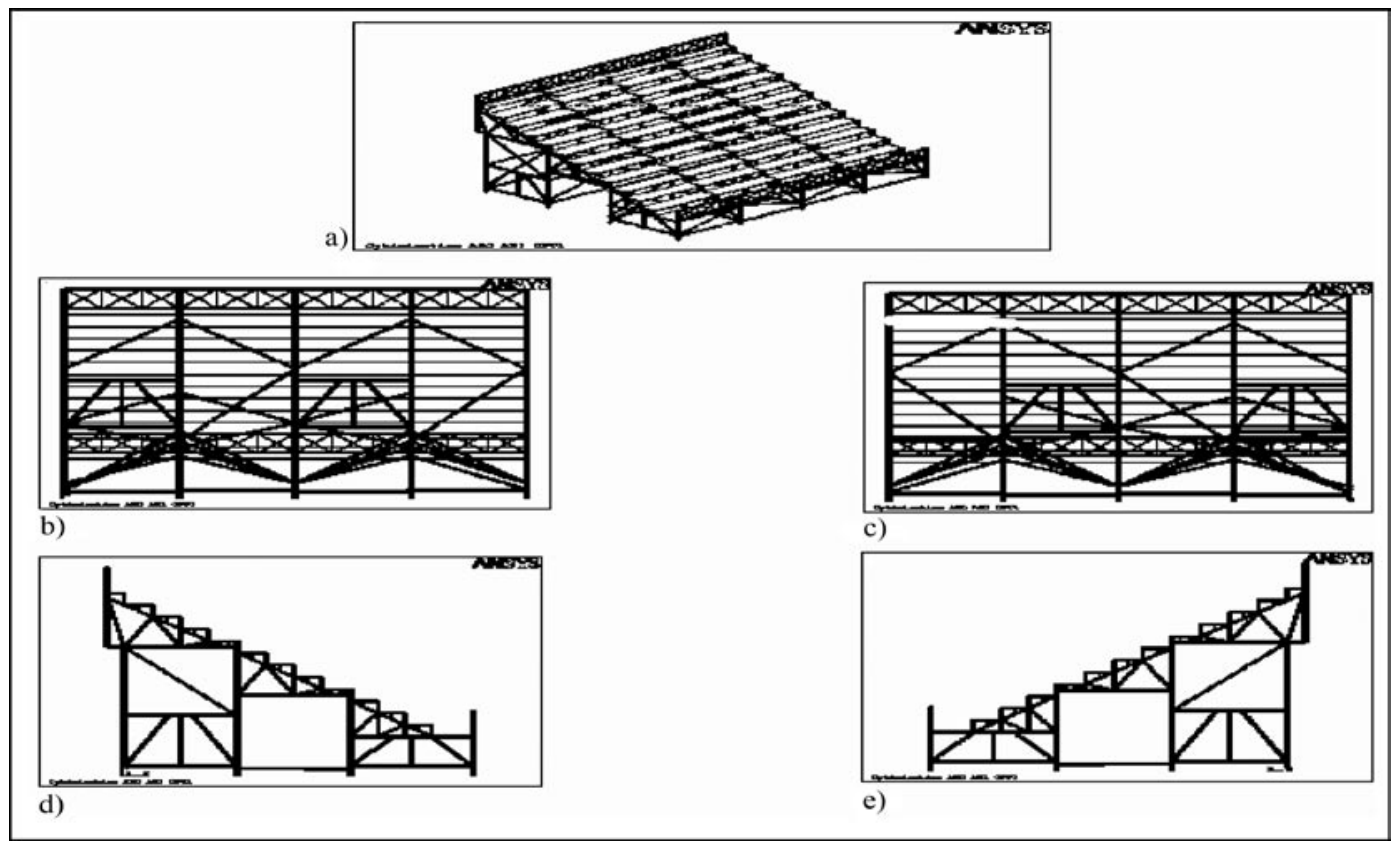

Figura 6: Estrutura AR1 - sistema de contraventamento otimizado: a) perspectiva, b) frente,

c) fundo, d) lateral esquerda e e) lateral direita.

\subsection{Otimização final da arquibancada AR1}

Apesar do aumento, a freqüência lateral ainda permaneceu abaixo da freqüência recomendada de $4,0 \mathrm{~Hz}$, que evitaria o risco de ressonância quando da aplicação das cargas dinâmicas induzidas por espectadores. Porém, a partir da estrutura obtida com a introdução do novo sistema de contraventamento, apesar do projeto continuar inviável, a estrutura tornou-se possível de ser viabilizada, conforme se verificou ao aplicar os procedimentos de otimização.

Os valores iniciais, os limites de projeto incluindo valores comercialmente disponíveis, além dos valores ótimos obtidos estão apresentados na Tabela 4. 
Tabela 4: Valores iniciais, valores limites e ótimos - AR1

\begin{tabular}{ccccc}
\hline \multirow{2}{*}{$\begin{array}{c}\text { Variáveis de Projeto/ } \\
\text { Parâmetros }\end{array}$} & \multirow{2}{*}{ Valores Iniciais } & \multicolumn{2}{c}{ Limites Comerciais } & \multirow{2}{*}{ Valores ótimos } \\
\cline { 3 - 4 } $\mathrm{d}_{1}(\mathrm{~mm})$ & 50,80 & 38,10 & 63,50 & 63,50 \\
$\mathrm{e}_{1}(\mathrm{~mm})$ & 3,0 & 2,0 & 4,25 & 3,0 \\
$\mathrm{~d}_{2}(\mathrm{~mm})$ & 38,10 & 33,50 & 50,80 & 50,80 \\
$\mathrm{e}_{2}(\mathrm{~mm})$ & 3,0 & 2,0 & 3,75 & 3,75 \\
$\mathrm{~d}_{3}(\mathrm{~mm})$ & 37,75 & 25,40 & 50,80 & 50,80 \\
$\mathrm{e}_{3}(\mathrm{~mm})$ & 3,0 & 2,0 & 3,75 & 2,0 \\
$\mathrm{~d}_{4}(\mathrm{~mm})$ & 12,70 & 12,70 & 31,75 & 12,70 \\
$\mathrm{e}_{4}(\mathrm{~mm})$ & 1,5 & 2,0 & 3,0 & 3,0 \\
$\mathrm{e}_{\mathrm{b}}(\mathrm{mm})$ & 20 & - & - & 20 \\
SMAX $(\mathrm{MPa})$ & 163 & - & 94,90 & 94,10 \\
FREQ $(\mathrm{Hz})$ & 2,36 & 4 & 7 & 4,85 \\
massa total $(\mathrm{kg})$ & 2893,1 & - & - & 3679,9 \\
\hline
\end{tabular}

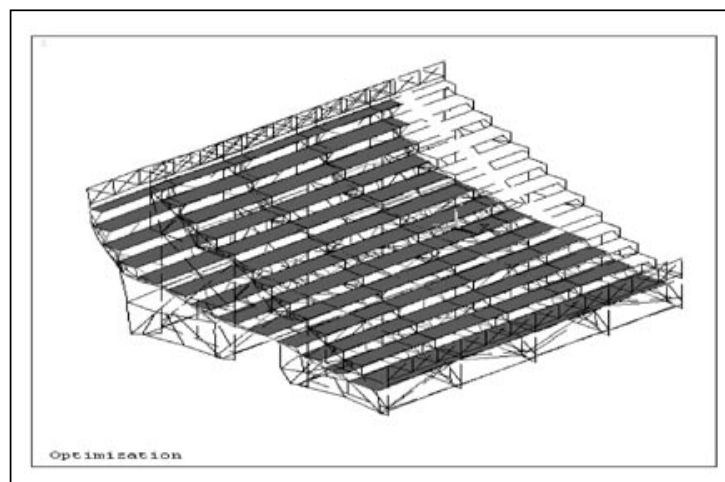

\begin{tabular}{|c|c|}
\hline Modos de vibração & Freqüências $(\mathrm{Hz})$ \\
\hline 1으 (lateral) & 4,85 \\
\hline 2o (frente-para-trás) & 7,21 \\
\hline $3^{\circ}$ (lateral) & 7,59 \\
\hline 4 o (frente-para-trás) & 9,22 \\
\hline 5으 (vertical) & 10,54 \\
\hline
\end{tabular}

Figura 7: Primeiro modo e freqüências naturais da estrutura AR1 após a otimização.

Nesta Tabela, as variáveis de estado SMAX e FREQ também são, respectivamente, os limites de tensão nas extremidades da barras e freqüência fundamental da estrutura, restrições estas relacionadas às Eqs. (4) e (5). A função objetivo é massa total da estrutura. O limite superior é $7,0 \mathrm{~Hz}$ na freqüência fundamental foi definido apenas para atender à exigência do algoritmo de otimização, conforme já explicado. $\mathrm{O}$ modo de vibração relacionado à freqüência fundamental pode ser visto na Fig. 7. No modelo otimizado, haviam 972 elementos e 530 nós. A reestruturação do sistema de contraventamento resultou em um aumento de 12 barras em comparação com a arquibancada original. No que diz respeito às restrições de freqüência natural, a aplicação dos cinco critérios propostos por Ji e Ellis (1997) resultou em uma estrutura possível de ser otimizada.

Deve-se notar que a massa total da estrutura aumentou, em decorrência da inclusão de novas barras e para satisfazer as restrições em termos dos níveis de tensão. Portanto, as dimensões finais obtidas para as barras estão relacionadas a uma condição de massa mínima da estrutura que satisfaça os critérios de projeto. As seções transversais 
dos elementos de barras tubulares tiveram suas áreas alteradas de $4,5 \mathrm{~cm}^{2}, 3,4 \mathrm{~cm}^{2}, 2,7 \mathrm{~cm}^{2}$ e $0,5 \mathrm{~cm}^{2}$ para os valores de $5,9 \mathrm{~cm}^{2}, 5,5 \mathrm{~cm}^{2}, 5,9 \mathrm{~cm}^{2}$, e $0,9 \mathrm{~cm}^{2}$, respectivamente. Sendo assim, apenas foi possível aplicar os procedimentos de otimização de forma eficiente após realizar as adequadas alterações no sistema de contraventamento, através da aplicação dos critérios discutidos.

\section{Conclusões}

Duas arquibancadas metálicas temporárias foram inspecionadas e serviram como casos de estudo nesta investigação. O módulo de otimização do software de análise em elementos finitos ANSYS (2001) foi aplicado com sucesso na otimização do projeto destas arquibancadas.

O emprego de análise por elementos finitos e do algoritmo de otimização foram suficientes para otimizar a arquibancada designada por MC1, pois neste caso a análise modal revelou que as freqüências naturais estavam fora de faixas críticas, sendo possível apenas através da otimização adequar o projeto estático para as limitações de tensão e freqüência natural. Por outro lado, para a estrutura designada por AR1, ao se aplicar o módulo de otimização do ANSYS, verificou-se que foi impossível obter um projeto viável, apesar das mudanças nas variáveis de projeto. Somente uma mudança na concepção do projeto que pudesse elevar a freqüência natural lateral da estrutura pôde conduzir a um projeto que atendesse às restrições em termos de freqüência. Isso foi possível introduzindo e reposicionando barras do sistema de contraventamento de modo a propiciar um acréscimo de rigidez lateral. Portanto, no estudo da arquibancada AR1, o foco das alterações foi o sistema estrutural de contraventamento e cinco critérios propostos na literatura para posicionar barras de contraventamento em pórticos planos foram utilizados com sucesso na estrutura tri-dimensional da arquibancada.

O procedimento empregado, mesclando análise em elementos finitos, regras para a disposição do contraventamento e técnicas de otimização, revelou-se uma alternativa prática para atender os requisitos estáticos e dinâmicos no projeto de arquibancadas temporárias. 


\section{Referências}

ANSYS User's Manual (2001). Revision 6.0.

Associação Brasileira de Normas Técnicas - ABNT (1980). Cargas para o Cálculo de Estruturas de Edificações. NBR 6120/80. Brasil.

Associação Brasileira de Normas Técnicas - ABNT (1986). Projeto e Execução de Estruturas de Aço de Edifícios. NBR 8800/86. Brasil.

Associação Brasileira de Normas Técnicas - ABNT (1997). Projeto de Estruturas de Madeira. NBR 7190/97. Brasil.

Haftka, R.T. and Gurdal, Z. (1993). Elements of Structural Optimization, 3rd edition. Kluwer Academic Publishers.

Ji, T. and Ellis, B.R. (1997). Effective Bracing Systems for Temporary Grandstands. The Structural Engineer, v. 75, n. 6, p. 95-100.

Ji, T. and Ellis, B.R. (1999). The Evaluation of Sports Stadia Grandstands for Dynamic Crowd Loads at Pop Concerts in the Unit Kingdom. Structural Dynamics - EURODYN' 99, Rotterdam, p. 937-942.

Littler, J.D. (1996). Measuring the Dynamic Response of Temporary Grandstands. Structural Dynamics - EURODYN'96, Rotterdam, p. 907-913.

Marinho, I.J.P. (2002). Projeto Ótimo de Estruturas de Arquibancadas Metálicas Reutilizáveis via ANSYS. Dissertação de Mestrado, Departamento de Engenharia Civil, Pontifícia Universidade Católica do Rio de Janeiro, Brasil.

More, J.J. and Wright, S.J. (1993). Optimization Software Guide, SIAM, Philadelphia, p. 13. 


\title{
Optimization of temporary grandstands through finite element analysis
}

\begin{abstract}
Studies of design optimization of temporary steel grandstands were carried out, taking as cases studies two inspected structures. Finite element models of the structures were developed and optimization routines were employed using the computational code ANSYS. The high stress levels in some bars due to static loads and the potential resonance of one of the structures in the lateral direction were the main problems identified. The bracing system was investigated as a way to avoid that natural frequencies of the structure were inside the critical frequency range in the lateral direction, which in turn was related to movements of the spectators in this direction. In the optimization process, the stress level and the fundamental natural frequency in the lateral direction were defined as state variables, the dimensions of the cross sections of the bars as design variables and the total mass of the structure as the objective function. It was found that one of the grandstands presented bars with cross section dimensions insufficient to resist the static loads. As for the other grandstand, in addition to the same problem with static loads, it also presented potential resonance in the lateral direction. In this case, the optimum design was only reached after rearranging bars of the bracing system.
\end{abstract}

Keywords: temporary steel grandstands, optimization, vibration, bracing system.

\section{Development}

Two temporary steel grandstands were inspected and taken as case studies, being called here as MC1 and AR1 (Fig. 1). The structures were modeled using the computational code ANSYS (Fig. 2). Static analyses were carried out aiming to identify structural elements with possible design problems. Modal analyses were subsequently conducted to determine values of the natural frequencies and mode shapes of the structures in order to identify the modes related to vibration in the lateral direction (Figs. 3 and 4). References in the literature indicate that, for the case of temporary grandstands, the main vibration problem is in the lateral direction and the respective natural frequencies should be above $4 \mathrm{~Hz}$ to avoid potential problems of resonance. Both structures were optimized by applying an algorithm available on the program ANSYS. In the optimization, the fundamental lateral natural frequency of the structure and the stress levels in the bars were defined as state variables, the dimensions of the cross sections of the bars as design variables and the total mass of the structure 
as the objective function (Tabs. 2 and 4, Fig. 7). In parallel, rules to place the bars of the bracing system (Fig. 6) were discussed and their effects in obtaining an optimum design for the grandstand AR1 analyzed.

\section{Discussion and Conclusions}

The ANSYS optimization algorithm was applied successfully to improve the static and dynamic design of the investigated temporary grandstands. For the grandstand $\mathrm{MC1}$, the modal analysis revealed that the natural frequencies were out of the critical frequency range of the excitation produced by spectators. Therefore, the optimization was focused on the static behavior. It was found that the structure presented bars with cross section dimensions insufficient to resist the imposed loads. As a result, the total mass of the structure was increased after optimization. The proposed modifications in the structure were: the areas of the cross sections of the steel hollow bars that presented values of $3,3 \mathrm{~cm}^{2}$ and $2,7 \mathrm{~cm}^{2}$ were changed to $4,8 \mathrm{~cm}^{2}$ and $1,6 \mathrm{~cm}^{2}$, respectively.

On the other hand, for the structure named AR1, initial results showed that it was not possible to reach an optimum design by applying the optimization algorithm, in spite of changes in the design variables. A need to change the conceptual design of the structure was found necessary in order to increase its lateral natural frequency prior to optimization.

The bracing system was the target system to be modified in order to increase the lateral natural frequencies of this grandstand. Its final optimized model presented 972 elements and 530 nodes. The rearrangement and addition of bars of the bracing system increased in 12 the number of bars in comparison with the original design. It should be noted that the change in the bracing system followed rules for placing bars presented in the literature for plane frames, which were investigated and applied successfully to the 3-D grandstand structure. The final dimensions obtained for the bars were related to the condition of minimum mass of the structure to satisfy the design criteria. The cross sections of the steel hollow bars had their areas of $4,5 \mathrm{~cm}^{2}, 3,4 \mathrm{~cm}^{2}, 2,7 \mathrm{~cm}^{2}$ and $0,5 \mathrm{~cm}^{2}$ altered to $5,9 \mathrm{~cm}^{2}, 5,5 \mathrm{~cm}^{2}, 5,9 \mathrm{~cm}^{2}$, and $0,9 \mathrm{~cm}^{2}$, respectively.

The overall procedure, by mixing finite element analysis, rules for placing bars in the bracing system and optimization techniques, was revealed to be a practical way to help designing temporary steel grandstands considering both the static and the dynamic requirements. 